\title{
Lung Abscesses in 2 Patients With Lancefield Group F Streptococci (Streptococcus milleri Group)
}

\author{
Vijaya K Gogineni MD and Ariel Modrykamien MD
}

\section{Introduction}

Lung abscess presents as a circumscribed accumulation of pus in the lungs, which is more common in patients predisposed to aspiration, immunosuppressed patients, and patients with chronic lung disease, malignancies, or diabetes mellitus. ${ }^{1}$ Lung abscess is also associated with dental caries, alcohol abuse, and uncontrolled epilepsy. Many reports associate lung abscess with the presence of anaerobic infection, ${ }^{1}$ but aerobic infections have been reported as well. Despite numerous descriptions of body abscesses produced by Streptococcus milleri group bacteria, lung abscess caused by this group remains under-reported. We present 2 patients admitted with lung abscesses produced by $S$. milleri group bacteria.

\section{Case 1}

A 64-year-old white woman with gastroesophageal reflux and inactive shingles presented with progressive dyspnea and left-side pleuritic chest pain for a week. She complained of progressive shortness of breath associated with intense pleuritic pain, low-grade fever, chills, and productive cough. She denied hemoptysis or weight loss. She was a former smoker of 60 packs of cigarettes per year. She had quit the nicotine habit 25 years prior. Notably, she had a root canal treatment 4 weeks prior to the admission. She presented with mild respiratory distress, tachypnea, and fever. Her $\mathrm{S}_{\mathrm{pO}_{2}}$ was $92 \%$ on supplemental oxygen ( $2 \mathrm{~L} / \mathrm{min}$ ) via nasal cannula. Other vital signs were within normal limits. Auscultation revealed decreased

The authors are affiliated with the Division of Pulmonary, Sleep, and Critical Care Medicine, Creighton University School of Medicine, Omaha, Nebraska.

The authors have disclosed no conflicts of interest.

Correspondence: Ariel Modrykamien MD, Division of Pulmonary, Sleep, and Critical Care Medicine, Creighton University School of Medicine, 601 N 30th Street, Suite 3820, Omaha NE 68131.

DOI: $10.4187 /$ respcare.01316 breath sounds, and fine crackles at the left chest base. The rest of the physical examination was unrevealing. Complete blood count showed a white cell count of 19,700 cells $/ \mu \mathrm{L}$ with a differential of $85 \%$ neutrophils. Comprehensive metabolic panel and coagulation panel were unremarkable. Enzyme-linked immunosorbent assay for human immunodeficiency virus was negative, as was sputum acid-fast bacilli test. Chest radiograph revealed left lower lobe infiltrate (Fig. 1). Computed tomogram (Fig. 2) revealed a well circumscribed mass in the posterior segment of the left lower lobe, consolidation in the lingula, and mediastinal and left hilar lymphadenopathies.

Based on the suspicion of lung malignancy (versus lung abscess), we conducted bronchoscopy and endobronchial ultrasound-guided transbronchial needle biopsy. Lymph node cytology was negative for malignancy. Bronchoalveolar lavage, bronchial brush cytology, and transbronchial biopsies of the left lower lobe mass were negative for malignancy. Bronchoalveolar lavage fluid and tissue cultures grew $S$. constellatus. Transthoracic needle biopsy of the heterogeneous consolidation of the lingula grew the same organism.

The diagnosis was lung abscesses secondary to $S$. constellatus. She was treated with ampicillin and sulbactam for 6 weeks and had substantial symptom improvement (Fig. 3).

\section{Case 2}

A 60-year-old male Burmese refugee with no noteworthy medical history was admitted with progressive shortness of breath and intermittent fever of 2 weeks duration. He started with the aforementioned symptoms, which were associated with chills and productive cough with yellowish purulent sputum. He denied hemoptysis, pleuritic pain, or weight loss. He was a current 25-pack-year smoker, for 45 years.

On examination he was in mild respiratory distress and febrile $\left(38^{\circ} \mathrm{C}\right)$. His blood pressure was $89 / 55 \mathrm{~mm} \mathrm{Hg}$ and his respiratory rate was 24 breaths/min. $\mathrm{S}_{\mathrm{pO}_{2}}$ was $90 \%$ on supplemental oxygen $(5 \mathrm{~L} / \mathrm{min})$ via nasal cannula. He had dental caries. There were decreased breath sounds with 


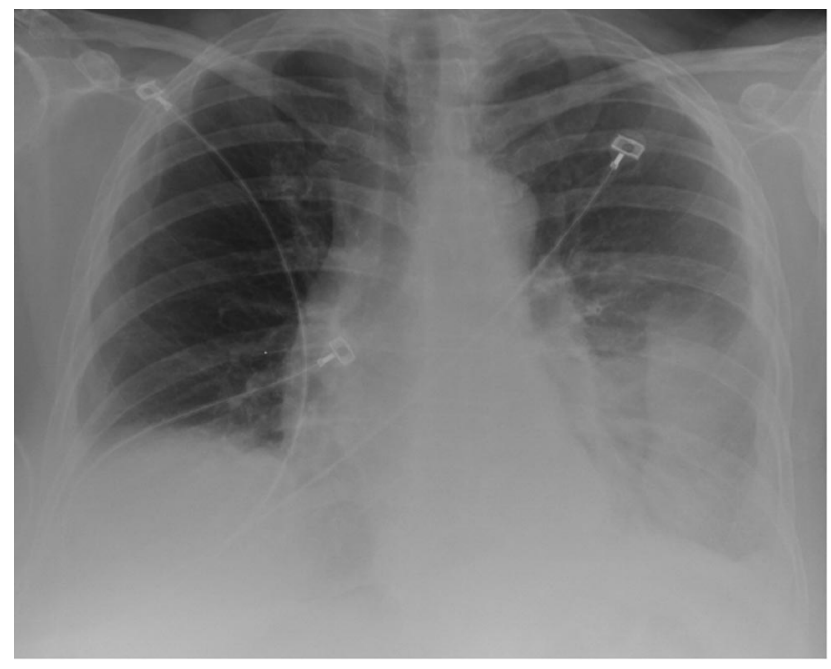

Fig. 1. Chest radiograph shows left lower lobe infiltrate.

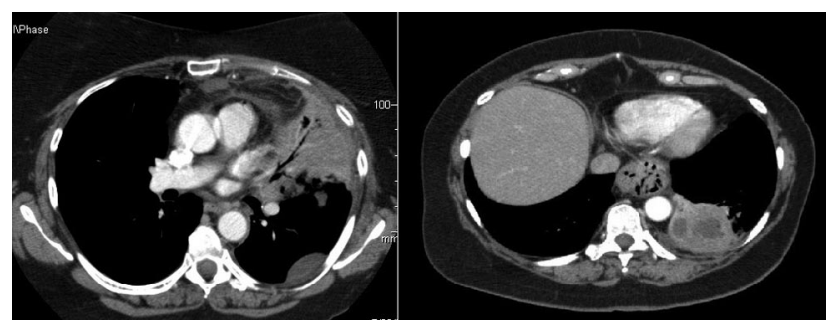

Fig. 2. Computed tomogram (mediastinal window) reveals a well circumscribed mass in the posterior left lower lobe and consolidation in the lingula.

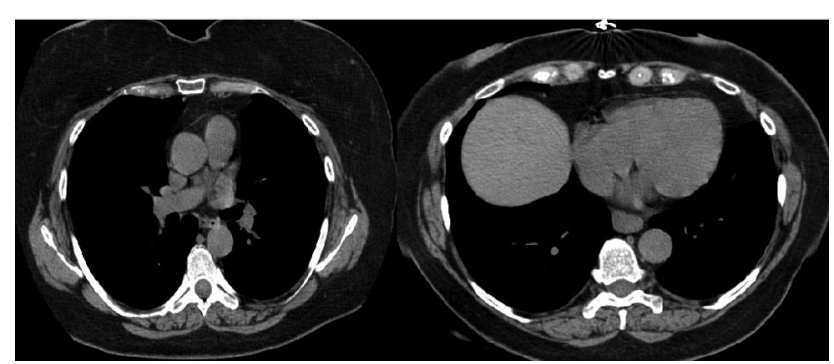

Fig. 3. Computed tomogram (mediastinal window) 2 months after finishing treatment shows complete resolution of lingular consolidation and left lower lobe abscess.

fine crackles in the left base. The rest of his physical examination was normal. Complete blood count was normal. The sodium concentration was $128 \mathrm{mEq} / \mathrm{L}$, and serum potassium was $2.8 \mathrm{mEq} / \mathrm{L}$. His renal and liver function tests were within normal limits. An adrenocorticotropic hormone stimulation test (with $250 \mu \mathrm{g}$ of adrenocorticotropic hormone) confirmed the diagnosis of primary adrenal insufficiency: the cortisol levels before and after the test were $17 \mu \mathrm{g} / \mathrm{dL}$ and $20 \mu \mathrm{g} / \mathrm{dL}$, respectively. Chest radiograph showed a left lower lobe infiltrate (Fig. 4).

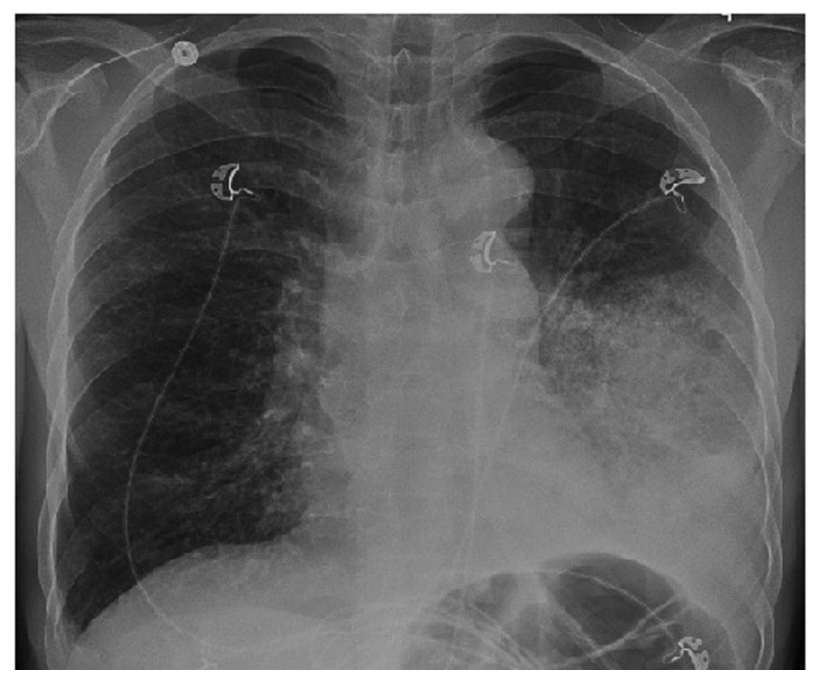

Fig. 4. Chest radiograph shows infiltrate in the left lower lobe.

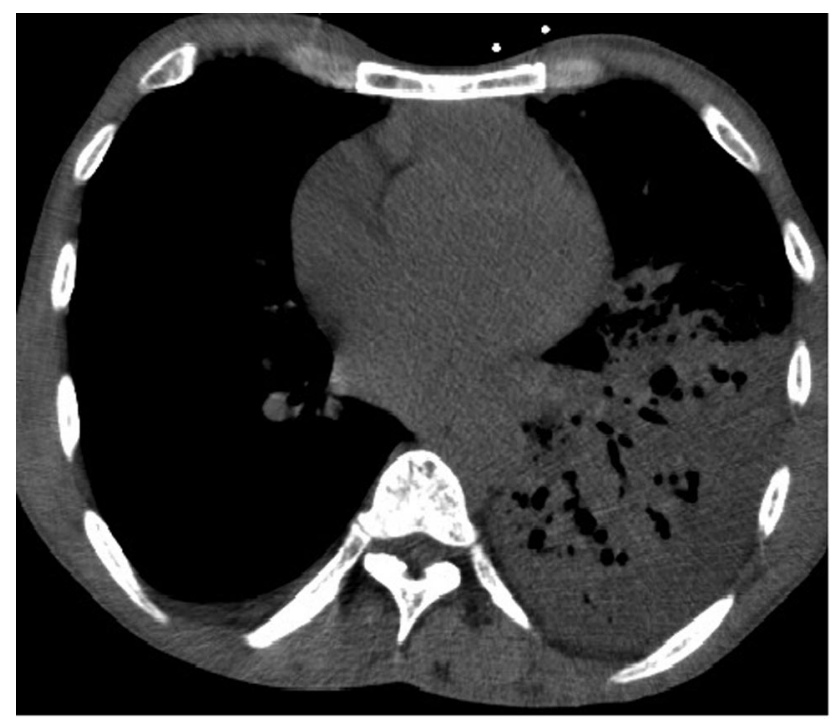

Fig. 5. Computed tomogram (mediastinal window) shows a consolidation with evolving cavitations in the left lower lobe.

Computed tomogram showed a consolidation with evolving cavitations in the left lower lobe (Fig. 5). Bronchoscopy with bronchoalveolar lavage and transbronchial biopsy was performed. Enzyme-linked immunosorbent assay for human immunodeficiency virus, sputum for acid-fast bacilli test, and blood cultures were negative. The bronchoalveolar lavage fluid grew Lancefield group F streptococci. The diagnosis was lung abscess secondary to Lancefield group F streptococci. He received ampicillin and sulbactam for 4 weeks. He also received intravenous hydrocortisone for adrenal insufficiency. His symptoms improved significantly. A repeat radiograph 6 weeks after completion of treatment showed substantial improvement (Fig. 6). 


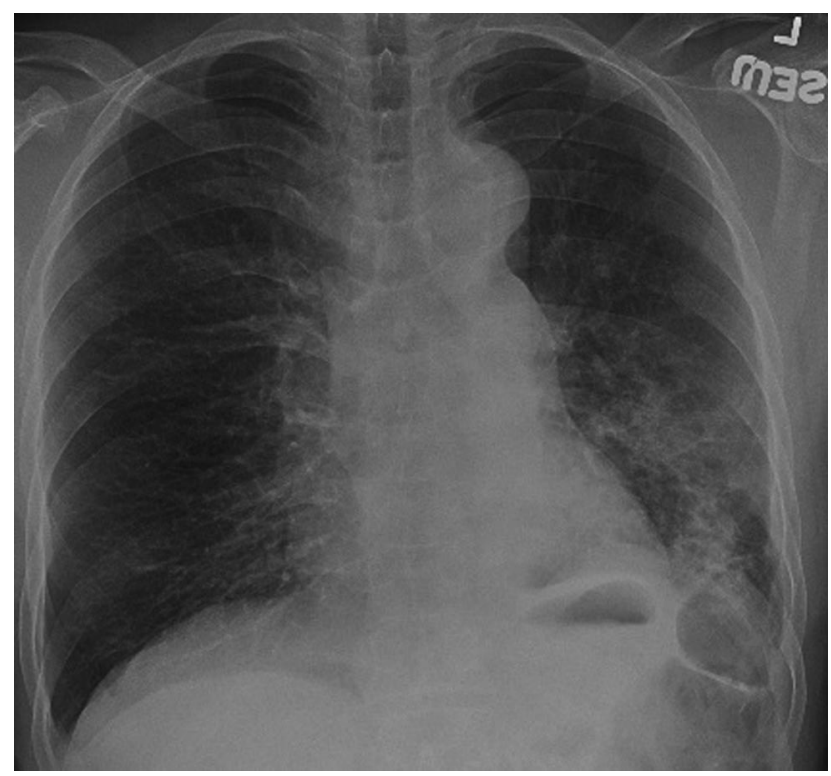

Fig. 6. Chest radiograph shows improvement of the left lower lobe infiltrate.

\section{Discussion}

Lung abscess most frequently arises as a complication of aspiration pneumonia, caused by mouth anaerobes such as Peptostreptococcus, Bacteroides, and Fusobacterium species. Aerobic bacteria that infrequently cause lung abscess include Staphylococcus aureus, S. pyogenes, S. pneumoniae, Klebsiella pneumoniae, Haemophilus influenzae, Actinomyces species, Nocardia species, and Gram-negative bacilli. ${ }^{2}$ Patients who develop lung abscess commonly have periodontal disease..$^{1,3} \mathrm{~A}$ bacterial inoculum from the gingival crevice reaches the lower airways, and infection is initiated because the bacteria are not cleared by the patient's host defense mechanisms. This results in aspiration pneumonitis and progression to tissue necrosis 7-14 days later, resulting in lung abscess. ${ }^{2,4}$ Lancefield group $\mathrm{F}$ and related streptococci ( $S$. milleri group) have emerged in recent years as organisms associated with purulent disease in humans. Group F streptococci are Grampositive, facultative anaerobic or capnophilic cocci that form pairs or chains. ${ }^{2}$ All group F streptococci belong to the broader $S$. milleri group, which includes 3 species: S. intermedius, S. anginosus, and S. constellatus. ${ }^{5}$ In 1977 , Facklam proposed to divide the isolates into 2 species, according to their capability to ferment lactose: S. milleri intermedius (lactose fermenting) and S. anginosus constellatus (unable to ferment lactose). ${ }^{6} \mathrm{~A}$ distinct but variable feature is the production of a caramel-like odor in culture. ${ }^{6}$ The $S$. milleri group is usually found among normal oropharyngeal and gastrointestinal flora. Among the most striking features of these species is their tendency to cause abscesses. ${ }^{6}$ From the entry site, these organisms can spread hematogenously to cause metastatic abscesses in distant organs. ${ }^{7}$ There have been reports of $S$. milleri group bacteria causing aortic-valve endocarditis, liver, ${ }^{8}$ brain, ${ }^{9}$ abdominal, ${ }^{10}$ and epidural abscesses, ${ }^{11}$ and even septic shock with bacteremia. ${ }^{12}$

Notably, a retrospective review of 25 cases of empyema, lung abscess, or both caused by $S$. milleri group bacteria revealed considerable mortality. The mouth and upper respiratory tract have frequently been regarded as the source of $S$. milleri group bacteria, and it is thought that infections are preceded by aspiration. ${ }^{13}$ Generally, $S$. intermedius and $S$. constellatus are more commonly associated with abscesses than is $S$. anginosus. S. intermedius also appears to cause more deep-seated abscesses than those due to S. constellatus. However, there were cases of septic shock due to $S$. constellatus bacteremia following tooth extraction. ${ }^{14}$ Abscesses caused by $S$. constellatus are more likely to be polymicrobial, with the co-isolate bacteria reflecting the site from which the specimen was obtained. ${ }^{15}$ All members of the $S$. milleri group are susceptible to $\beta$-lactams, and the usual dosage is utilized. ${ }^{15}$ Variable susceptibility to tetracycline, clindamycin, and erythromycin has been reported as well. ${ }^{5,15}$ Abscess drainage might be required in some cases. In fact, infections due to $S$. constellatus or $S$. anginosus have been treated with simple incision and drainage and antibiotics. Conversely, $S$. intermedius abscess usually requires extensive surgery or computed-tomography-guided biopsy for both therapeutic and diagnostic purposes. ${ }^{13}$ We treated our 2 patients conservatively with intravenous antibiotics, and both had complete clinical and radiologic resolution of the abscesses. In the first patient we chose the intravenous route because of lack of response to prior oral antibiotics. In the second patient we used the intravenous route because he had recurrent tonsillitis, which precluded consistent oral therapy.

\section{Teaching Points}

- S. milleri has increasingly been associated with serious purulent infection at various body sites, including the lung parenchyma.

- Invasive procedures such as bronchoscopy and/or transthoracic needle biopsy are required to confirm a diagnosis of $S$. milleri group abscess.

- Intravenous antibiotics for 4-6 weeks, and eventual surgical drainage, are essential to obtain a positive clinical outcome.

\section{REFERENCES}

1. Wong CA, Donald F, Macfarlane JT. Streptococcus milleri pulmonary disease: a review and clinical description of 25 patients. Thorax 1995;50(10):1093-1096. 
2. Wiedemann HP, Rice TW. Lung abscess and empyema. Semin Thorac Cardiovasc Surg 1995;7(2):119-128.

3. Shinzato T, Saito A. The Streptococcus milleri group as a cause of pulmonary infections. Clin Infect Dis 1995;21(Suppl 3):S238-S243.

4. Schiza S, Siafakas NM. Clinical presentation and management of empyema, lung abscess and pleural effusion. Curr Opin Pulm Med 2006;12(3):205-211.

5. Whitworth JM. Lancefield group F and related streptococci. J Med Microbiol 1990;33(3):135-151.

6. Facklam RR. Physiological differentiation of viridans streptococci. J Clin Microbiol 1977;5(2):184-201.

7. Whiley RA, Beighton D, Winstanley TG, Fraser HY, Hardie JM. Streptococcus intermedius, Streptococcus constellatus, and Streptococcus anginosus (the Streptococcus milleri group): association with different body sites and clinical infections. J Clin Microbiol 1992; 30(1):243-244.

8. Rashid RM, Salah W, Parada JP. 'Streptococcus milleri' aortic valve endocarditis and hepatic abscess. J Med Microbiol 2007;56(Pt 2): 280-282.

9. Jan F, Hafiz AM, Gupta S, Meidl J, Allaqaband S. Brain abscesses in a patient with a patent foramen ovale: a case report. J Med Case Reports 2009;3:9299.
10. Silderhuis VM, Zijlstra JG. Abdominal abscesses with Streptococcus milleri group after laparoscopic chromopertubation. Acta Obstet Gynecol Scand 2010;89(7):982-983.

11. Lampen R, Bearman G. Epidural abscess caused by Streptococcus milleri in a pregnant woman. BMC Infect Dis 2005;5:100.

12. Ng KW, Mukhopadhyay A. Streptococcus constellatus bacteremia causing septic shock following tooth extraction: a case report. Cases J 2009;2:6493.

13. Claridge JE 3rd, Attorri S, Musher DM, Hebert J, Dunbar S. Streptococcus intermedius, Streptococcus constellatus, and Streptococcus anginosus ("Streptococcus milleri group") are of different clinical importance and are not equally associated with abscess. Clin Infect Dis 2001;32(10):1511-1515.

14. Bantar C, Fernandez Canigia L, Relloso S, Lanza A, Bianchini H, Smayevsky J. Species belonging to the "Streptococcus milleri" group: antimicrobial susceptibility and comparative prevalence in significant clinical specimens. J Clin Microbiol 1996;34(8):20202022.

15. Jacobs JA, Pietersen HG, Stobberingh EE, Soeters PB. Bacteremia involving the "Streptococcus milleri" group: analysis of 19 cases. Clin Infect Dis 1994;19(4):704-713. 